\title{
Exclusión y conocimiento social ${ }^{1}$
}

RAQUEL SOSA ELIZZAGA*

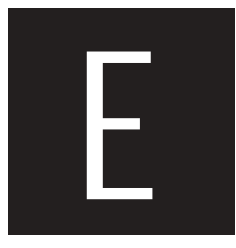

I combate a la pobreza se ha convertido en el mejor negocio del siglo que comienza, declaró un empresario mexicano recientemente. ${ }^{2}$ En efecto, a lo largo de los últimos diez años, cientos de miles de dólares - tal vez millonesse han gastado en investigaciones realizadas en el mundo para determinar la evolución de la pobreza, sus características y, sobre todo, sus riesgos. El Banco Mundial asoció hace una década la pobreza a la delincuencia, y más recientemente, afirmó que las crisis económicas, aunadas a la destrucción de las instituciones de mediación, conforman hoy en el mundo un escenario amenazante, toda vez que la incertidumbre obliga a comportamientos inesperados por parte de sociedades empobrecidas y desesperadas. $^{3}$

Por su parte, la estadística social se ha transformado con los años para convertirse en un sofisticado instrumento de políticas llamadas sociales cuyos objetivos han ido de la pretensión de incluir a los pobres en el mercado, a la contención de los movimientos de pobres vía esquemas de ayuda focalizada a zonas potencialmente críticas. Preguntarse -e investigar- cuántos

\footnotetext{
* Profesora de tiempo completo de la Facultad de Ciencias Políticas y Sociales de la U niversidad N acional Autónoma de México. Socióloga y Doctora en Historia. México.

1 Ponencia presentada en la Primera Conferencia Regional de la Asociación Internacional de Sociología en América Latina, Porlamar, Isla de Margarita, Venezuela, 7 al 11 de mayo de 2001.

2 Carlos Slim, multimillonario dueño del consorcio Carso, La Jornada, 24 de abril del 2001.

3 Banco Mundial (dos estudios).
} 
son los pobres y dónde se encuentran parece ser el reto más novedoso y rentable para los políticos, funcionarios y especialistas.

No obstante, el éxito de tales empeños ha sido dudoso, tanto desde el punto de vista del mercado, como del control de los pobres de carne y hueso. Y quiero señalar sólo dos ejemplos: la estadística sobre la evolución de la fuerza de trabajo ha sido abundante, pero no ha logrado avanzar en la distinción de empleo y desempleo. Los parcial o temporalmente ocupados, quienes no pagan impuestos, quienes no cotizan en el sistema de seguridad social, quienes reciben salarios inferiores a dos dólares diarios (considerado como indicativo de pobreza extrema) siguen siendo un misterio a dilucidar.

No basta con afirmar, como se hizo hace unosaños, que los pobres se dedican a actividades "informales" (cualquiera que observe a los vendedores del centro histórico de la ciudad de M éxico puede, por lo demás, darse cuenta rápidamente que son todo menos eso). Tampoco es suficiente pretender captarlos para el mercado con la adopción de medidas extremas, como la que hoy ocupa al gobierno mexicano de cobrar impuestos a alimentos básicos y medicinas. Su presencia organizada es visible en todas nuestras ciudades y constituye un motivo de alarma policiaca. El hecho es que no hay hasta ahora quien pueda afirmar que dispone de alternativas que no sea la de la "tolerancia cero" - a su peculiar y compleja lucha por la sobrevivencia. ${ }^{4}$

Y vamosal segundo ejemplo: la determinación de dónde se encuentran los pobres ha provocado un largo debate en las ciencias sociales, la estadística y la política en nuestros países. Durante años, y pese a todas las evidencias en contrario, las políticas de combate a la pobreza se orientaron a "privilegiar" a aquellas comunidades rurales cuyas características podían ser incluidas en un tipo ideal: las que sufren de analfabetismo, habitan en viviendas 
precarias, carecen de servicio de agua potable o energía eléctrica y tienen salarios inferioresal mínimo determinado en cada país.

En M éxico, semejante visión condujo a excluir hasta el día de hoy de los programas de combate a la pobreza a los pobres urbanos, y particularmente, a los habitantes de las ciudades más grandes. ${ }^{5}$ U na vez más, cualquier observador puede confirmar - sin que por ello tenga pretensiones de cientificidad- a esos pobres urbanos que, sin ser analfabetas (en la ciudad de M éxico, por ejemplo, el promedio de estudios es de 9 años); sin sufrir la carencia de energía eléctrica ( $98 \%$ de los habitantes de la ciudad tienen, de uno u otro modo este servicio) o agua (aunque cientos de miles reciban agua en pipas, por tandeo, o de pésima calidad); sin habitar en una vivienda con piso de tierra (las barrancas, cerros y aún las vecindades del centro de la ciudad de México no tienen esas características), no puede considerárseles de otro modo que pobres, y extremadamente pobres.

Mientras tanto, cientos de comunidades rurales convertidas por la pobreza en pueblos fantasmas, o habitadas exclusivamente por mujeres, dan lugar a la existencia de pequeños centros comerciales, casas de cambio, circulación de dinero, camionetasy autos modernos, cambio repentino de hábitos y vida estacional ajena a su tradicional tranquilidad. Todo ello ocurre como consecuencia del ir y venir de trabajadores migrantes que otra vez, contra todo despliegue militar y policiaco- cruzan una y otra vez la frontera en busca de mejorar sus ingresos y la calidad de vida de sus familias. ${ }^{6}$

Así, cuando alguien se empeña sinceramente en comprender dónde están, quiénes son, de qué carecen efectivamente los pobres, la literatura sobre ellos y la política con que se les trata parece un sinsentido, un discurso vacío de contenido. El conocimiento que se tiene sobre este fenómeno social, el más visible, el más presuntamente reconocido en nuestro tiempo 
es pobre, inexacto, y ayuda poco, si de lo que se trata es de llevar a cabo algo más que una tarea cosmética.

\section{Eppur, si muoven...}

Un esfuerzo de otra naturaleza se ha llevado a cabo entre las organizaciones civiles, sociales, los partidos democráticos y el pensamiento crítico. A través de los años de las posdictaduras y posguerras, han sido sobre todo ellos quienes se han sensibilizado a la necesidad de red efinir sus perfiles y su relación con quienes sufren, simultáneamente, por la pobreza, la injusticia y la constante violación a sus derechos. En América Latina, la multiplicación de iniciativas de acción colectiva, el abandono de las pretensiones de vanguardia y el desarrollo de experiencias alternativas de gestión gubernamental han dado como resultado una visión compleja aunque no siempre articulada- de las demandas y necesidades de las más de cuatrocientos millones de personas que se encuentran en condiciones de pobreza.

Es posible que sea insuficiente la reflexión sobre cómo se ha modificado y cuáles siguen siendo asuntos sin resolver en la relación entre la política, los políticos y las organizaciones reales de esa parte de la llamada sociedad civil. N o obstante, podemos afirmar que ninguna asociación política o gobierno en el subcontinente ha sido ajena a fuertes convulsiones y cambios a partir del impulso y la inobjetable presencia de los desposeídos. Y para muestra están la organización del M ovimiento de los Sin Tierra en el Brasil; el movimiento indígena en el Ecuador y en México; el movimiento de los campesinos cocaleros en Colombia y Bolivia; el peso de los migrantes en la determinación de políticas en México y Centroamérica; los movimientos por los derechos humanos en Chile y Argentina.

La insuficiencia e inconsecuencia de las políticas de combate a la pobreza se han hecho en primera instancia evidentes mediante la acción 
organizada de los propios desposeídos. $\mathrm{H}$ an sido ellos quienes han denunciado la manipulación de instituciones y programas; el efecto que crean políticas de focalización en la división de las comunidades; su conversión en políticas contrainsurgentes conocidas como de seguridad pública en cada uno de nuestros países. Los objetivos declarados de los programas han sido confrontados con su aplicación práctica. Las limitaciones y contradicciones en que han incurrido sus promotoresno han resultado sino en la agudización de la problemática que dicen buscar resolver.

Si la denuncia de semejante estado de cosas ha abierto un campo vastísimo de acción política a las organizaciones, no ha resuelto, sin embargo, el problema fundamental de articular un conocimiento social capaz de enfrentar - a partir de las condiciones actuales- la articulación de los desposeídos en la formulación y proyección de alternativas de tranformación en el sentido de la justicia social en el mediano y largo plazos, como tampoco la superación de las situaciones más dramáticas y urgentes.

\section{La pobreza como objeto de asistencia social}

La situación de los excluidos ha merecido, a lo largo de los años, una consideración ambigua de parte de los gobiernos y los grupos de poder. Por una parte, no cesa de haber la visión de que deben ser integradosal mercado: "Nada es gratis en esta vida", dijo recientemente el Presidente mexicano en defensa de su reforma fiscal. Q uienes nada tienen no valoran lo que se les ofrece si no se les cobra, es la razón que se esgrime cuando se incorporan cuotas a la educación, restricciones y procesos de privatización de losservicios de salud pública, etc. Mas esta visión no deja de acompañarse del reconocimiento de la necesidad de "ayudar", "aliviar", hacer menos dramática la situación de quienes sufren especialmente por la miseria en que viven, a quienes se considera en condiciones de vulnerabilidad. 
Así, se promueven apoyos temporales a asociaciones privadas que practican la caridad; se difunden campañas de ayuda a víctimas de desastres; se distribuyen dulces y juguetes en las fiestas. Los más pobres se vuelven entonces motivo de vergüenza pública, tema de encendidos discursos y hasta objeto del sincero dolor o preocupación por parte de quienes conocen su situación. No tanto, sin embargo, como para que se propongan políticas efectivas que les permita elevarse a la condición de ciudadanos. No tanto como para que pretendan ejercer y exijan el cumplimiento de sus derechos. Son objeto, no sujeto de políticas en su beneficio.

El terreno ocupado por la asistencia ha sido tradicionalmente negado por las organizaciones de izquierda y el pensamiento crítico. No hay en esta materia, la de quienes suman cientos de miles pero no necesariamente expresan en forma de lucha organizada sus demandas, una exigencia de conocimiento y responsabilidad colectiva que lleve a realizar investigaciones y a promover políticas alternativas. Es como si de algún modo coincidieran visiones encontradas en otros espacios, en que lo único que pueden lograrse son paliativos para enfrentar las que no son sino condiciones concretas de vida de los pobres.

Sólo recientemente se ha iniciado el debate y comienzan a tener visibilidad las demandasespecíficas de justicia social, de equidad, de tolerancia y respeto a la diversidad, de integración verdadera, que presentan estos grupos de excluidos: los adultos mayores abandonados; los niños que sufren maltrato, violencia o explotación; las mujeres solas, jefas de familia; las personas con discapacidad; los jóvenes excluidos de opciones de estudio y de trabajo; los enfermos terminales; las víctimas de desastres.

En el panorama de un empobrecimiento generalizado de la población, el conocimiento de problemáticas sociales específicas, de demandas sentidas, aunque ocultas, la búsqueda de una relación directa y efectiva con políticas que se orienten para su beneficio sigue siendo una tarea pendiente 
y, en gran medida, inatendida. La pretensión de homogeneizar-cuantificarpor la vía de la estadística y de la política de combate a la pobreza a quienes sufren situaciones o condiciones diversas de vulnerabilidad ha dado hasta ahora al traste con toda opción de acceder con efectividad a su compleja problemática. $^{7}$

\section{Pobreza y exclusión}

Las líneas de pensamiento que hemosesbozado difícilmente se cruzan. Desde una perspectiva, la pobreza es un fenómeno masivo, cuantificable, comprensible sólo con la lente de parámetros rígidos, sobre los que pueden aplicarse políticas cuya eficacia es -o debiera ser- medible en plazos temporales variables. Los residuos de lo que no puede ser captado con esa lente es, en esa visión, objeto de asistencia social. Al fin y al cabo, lo que interesa es identificar la extensión e intensidad de la acción combinada del mercado y del Estado, enfrentar y contener eventuales o reales expresiones de inconformidad.

El descubrimiento que han hecho quienes realizan una reflexión desde el punto de vista de los movimientos sociales es otra. Los movimientos de pobres no corresponden a las expectativas de la estadística o la política social, sino algo más complejo y difícil de asir, y que tiene que ver con la existencia real, sensible, de seres humanos diversos, con problemáticas y demandas específicas, en una palabra, con voluntad propia.

Las organizaciones civiles y sociales han descubierto hace años el fenómeno de la exclusión, que puede definirse como la condición que agrega a la pobreza el hecho de la imposibilidad de incorporarse con plenos derechos a la vida social, al ejercicio de la ciudadanía. M as esta condición

7 Cf. Discurso de Marcos frente a la Cámara o en la Universidad. 
no es, por los ejemplos que hemos expuesto, exclusivamente referida a una carencia, sino que se manifiesta en la exigencia de participación directa en las decisiones que el poder asume en presunto beneficio de la sociedad en su conjunto. ${ }^{8}$ En efecto, no se trata exclusivamente de la determinación de un objeto -la pobreza- y sus consecuencias en términos de las dificultades que presupone para realizar los mínimos de producción y reproducción de la vida humana. De lo que se trata es de reconocer una condición que incluye la actividad de quienes sufren de carencias, sus demandas, su organización, su visión del mundo.

Ser excluido no significa solamente ser pobre. Significa no haber sido considerado en la determinación de los asuntos públicos. Significa estar ausente de la política que define el rumbo de una sociedad en una época, en un territorio determinado. Y es en esa dirección que la mayor parte de los estudios sobre la pobreza son, no sólo insuficientes, sino peligrosamente sesgados: desconsideran la condición humana, que significa pensamiento, voluntad, decisiones, presiones, temores, hábitos, experiencias, memoria.

De otra parte, en el conocimiento de los movimientos sociales y de sus efectos en la actividad política muchos intelectuales críticos han puesto por delante la definición de los pobres como luchadores antisistémicos per se. Han supuesto, durante años, que la evolución en las formas de organización y la lucha por la supervivencia de millones de seres humanos se orientará más tarde o más temprano en el sentido de la transformación social progresiva.

U na parte de los intelectuales críticos confunde sus propias aspiraciones y deseos con una realidad que frecuentemente se orienta en sentido inverso. Movimientos de masa que sustentan opciones políticas conservadoras, promueven guerras, son excluyentes e intolerantes. El entusiasmo tempo- 
ral por los triunfos de masa de opciones de izquierda cede inesperadamente ante el avance de ideologías y prácticas completamente alejadas de los objetivos de transformación social orientados por la justicia, la solución pacífica de los conflictos, la construcción de una ciudadanía tolerante y diversa.

Un espacio se ha abierto para explicar cambios políticos y de pensamiento independientes al mejoramiento de las condiciones de vida de millones de seres humanos en el planeta: la estrategia discursiva de los medios de comunicación. Parte importante de esta estrategia-considerada la más exitosa de las políticas tendientes a la consolidación de un statu quo profundamente excluyente- ha sido la conformación de un modelo de ciudadano 0 , más precisamente, de consumidor potencialmente capaz de acceder a opciones de bienestar que van de la integración a la modernidad en general a la posibilidad específica de elegir bienes o servicios tangibles o intangibles. ${ }^{9}$

En la búsqueda de una hegemonía política basada en el mercado, la estrategia de comunicación basada en la homogeneización de patrones de comportamiento, de expectativas y valores colectivos ha cumplido un papel significativo. Es el correlato más exitoso de políticas de combate a la pobreza cuyos resultados son objetivamente pobres. Esta estrategia ha mostrado su eficacia en la manipulación de conciencias, en la formación de movimientos, en la aprobación o rechazo colectivos a comportamientos determinados de dirigentes, políticos, grupos empresariales y desde luego, planes militares.

Políticos inescrupulosos han utilizado desde hace muchos años esta opción que permite desconocer realidades específicas a cambio de conquistar a millones de adherentes a la esperanza de integración a un mundo de satisfactores determinados o indeterminados. El éxito de tales propuestas

9 Desde perspectivas distintas, el fenómeno ha sido analizado por García Canclini, Bolívar Echeverría, Pablo González Casanova, Edgardo Lander... 
se mide en encuestas y triunfos electorales; en incremento del consumo de determinados bienes o en la modificación de comportamientos masivos. Particularmente sensibles resultan quienes viven la incertidumbre de cambios vertiginosos en la vida social.

El límite de dichas formas de integración puede hacerse evidente en efecto retardado, pero es inevitable. La promesa de un bienestar futuro, del acceso a bienes de consumo, del olvido de condiciones traumáticas o precarias, cede en plazos cada vez más cortos a la constatación de insuficiencias reales, de falta de atención a los problemas de mayor profundidad, de negativas como respuesta a demandas masivas. La exclusión -ese fenómeno difícilmente cuantificable, pero objetivo- reaparece a la vuelta de cada período y se manifiesta como inconformidad, como exigencia, como frustración acumulada.

Vivimosactualmente lo que podría denominarse una suerte de anomia en la anomia, si asumimos que la pobreza no es sólo un dato técnicamente verificable, sino resultado de políticas estatales que han propiciado el deterioro en las condiciones y calidad de vida, traumáticas experiencias autoritarias, abandono acumulado, intolerancia y exclusión. La contrastación de la visión homogeneizadora, del pretendido pensamiento único con una realidad tercamente diversa, no ha sido, sin embargo, una operación quirúrgica de pensadores brillantes. Esproducto de la experiencia y la reflexión de quienes han sido capaces de transformar sus carencias en exigencia de respeto, ejercicio de derechos, identidad propia, dignidad.

Dignidad significa en primera instancia el reconocimiento de los seres humanos como complejos, plurales, diversos, con necesidades y demandas específicas, con visiones, responsabilidades e historia propias. Dignidad significa lucha por la supervivencia, condición de pobreza que no se resuelve con ayuda económica, con promesas de mejoramiento social, con programas masivos de pretensiones unificadoras. Y sólo a partir del reconocimiento 
de la dignidad como valor humano esencial es que puede plantearse una transformación profunda de los supuestos científicosy políticos con que se han tratado hasta ahora la pobreza y la exclusión.

\section{La dignidad de los excluidos}

Las ciencias sociales han sufrido hace años la confusión promovida desde el poder acerca de quiénes son y cómo se comportan los sujetos sociales. La proliferación de la pobreza, las transformaciones de la vida social, las formas de lucha por la supervivencia, han permanecido ajenas a la mayor parte de los investigadores y políticos que aplican programas orientados a colectividades diversas.

Escasos han sido quienes, desde la perspectiva histórica y social han reconocido la existencia de códigos de comportamiento colectivo que determinen, más allá de condiciones materiales de vida, de la existencia de una u otra forma de gobierno, de la política específica que se aplique sobre un grupo social, el movimiento de las sociedades en el proceso de su transformación. Búsquedas como las de Barrington M oore, Theda Skopcol, Charles Tilly, Eric Hobsbawm permanecen como esfuerzos relativamente solitarios, que debieran dar lugar a investigaciones y propuestas políticas y sociales de más largo alcance, en la perspectiva de construir una concepción articulada de los sujetos sociales contemporáneos. ${ }^{10}$

Algunas de las claves del conocimiento por ellos aportados se encuentran en el estudio específico de las causas y consecuencias de comportamientos políticos y sociales de los excluidos, particularmente en situaciones de crisis. Cómo se orienta su lucha por la supervivencia; qué determina el peso de valores presentes en la acción de diversos grupos 
sociales; cómo influyen acontecimientos específicos en la orientación de los que se convierten en movimientos; cómo se viven, porqué son tolerables, cuándo se tornan intolerableslas condiciones de pobreza, exclusión, opresión.

Es urgente que emprendamos en esa perspectiva la revisión de los criterios y procedimientos con que se ha enfrentado esta dimensión mayoritaria de la vida social, ya que no sólo no hemos sido hasta ahora capaces de entender cabalmente la situación en la que se encuentran, sino que objetivamente hemos menospreciado o desconocido su dignidad, sus aportaciones creativas y de conocimiento, su intervención en la vida social.

Las preguntas se multiplican y nuestro problema no es sólo -aunque siga siendo- quiénes son y dónde están los pobres y excluidos, sino cuáles son sus necesidades, cuáles nuestras responsabilidades en el contexto de la lucha por la supervivencia de la que participan esos millones de seres humanos a los que nuestras sociedades y -sobre todo- la mayor parte de nuestros go biernos han dejado fuera de toda opción de ejercer sus derechos.

La exterioridad pasa a ser un supuesto inadmisible, si de lo que se trata es de penetrar en el conocimiento y-ojalá- en el compromiso de transformación en sentido democrático y de justicia de las formas de vida mayoritarias en sociedades de pobres. Los dilemas de atraso o modernidad, civilización o barbarie, ellos y nosotros, deben ceder el lugar a la búsqueda de elementos que nos permitan situarnos como parte de sociedades reales, en que las carencias y demandas de la mayoría lo son, en estricto sentido, de todos.

Con esas premisas, el conocimiento del poder, del ejercicio de la política y del papel del Estado pueden ser emprendidos a partir de la interiorización de las formas que han adquirido la explotación, la opresión, la injusticia, la exclusión en nuestras sociedades. Y no puede ser ajeno a la comprensión de los comportamientos del conjunto de los sujetos que interactúan en nuestras sociedades: sea en su enfrentamiento con el mer- 
cado, con el Estado, con la política y los políticos, sea en la relación cotidiana de lucha por la supervivencia en cada uno de los espacios que ocupa.

Redimensionar las formas que adquiere la presencia social de los excluidos en su diversidad, sus expresiones contradictorias, sus exigencias, el ejercicio de sus derechos, es una de las grandes tareas de las ciencias sociales contemporáneas. Es una tarea que no admite modelos, parámetros o estructuras de pensamiento y catego rizaciones rígidas. Difícilmente puede pensarse como trabajo individual, pero en ningún caso puede ser emprendida si se parte de puntos de vista que no incorporen las relaciones espaciales y temporales, la transversalidad, el carácter y calidad de las confrontaciones, la dignidad de los sujetos de conocimiento.

En el aparentemente escaso margen que deja a la imaginación la precariedad de la vida de millones de seres humanos existen inmensas e inexploradas capacidades creativas, opciones de superación de los más graves problemas sociales, perspectiva de futuro, esperanza. Son los ellos que están en todos nosotros, los que no han sido vistos ni escuchados pero luchan a brazo partido por sobrevivir en condiciones adversas, quienes reciben y resignifican señales contradictorias procedentes de la sociedad, el mercado, la política reconocidas, quienes muestran el camino para conocer y actuar en la sociedad contemporánea. 


\section{Resumo}

Las ciencias sociales han sufrido hace años la confusión promovida desde el poder acerca de quiénes son y cómo se comportan los sujetos sociales. La proliferación de la pobreza, las transformaciones de la vida social, las formas de lucha por la supervivencia, han permanecido ajenas a la mayor parte de los investigadores y políticos que aplican programas orientados a colectividades diversas.

La estadística social se ha transformado con los años para convertirse en un sofisticado instrumento de políticas llamadas sociales cuyos objetivos han ido de la pretensión de incluir a los pobres en el mercado, a la contención de los movimientos de pobres vía esquemas de ayuda focalizada a zonas potencialmente críticas. Preguntarse -e investigar- cuántos son los pobres y dónde se encuentran parece ser el reto más novedoso y rentable para los políticos, funcionarios y especialistas.

Escasos han sido quienes, desde la perspectiva histórica y social han reconocido la existencia de códigos de comportamiento colectivo que determinen, más allá de condiciones materiales de vida, de la existencia de una u otra forma de gobierno, de la política específica que se aplique sobre un grupo social, el movimiento de las sociedades en el proceso de su transformación.

Redimensionar las formas que adquiere la presencia social de los excluidos en su diversidad, sus expresiones contradictorias, sus exigencias, el ejercicio de sus derechos, es una de las grandes tareas de las ciencias sociales contemporáneas. Es una tarea que no admite modelos, parámetros o estructuras de pensamiento y categorizaciones rígidas. Difícilmente puede pensarse como trabajo individual, pero en ningún caso puede ser emprendida si se parte de puntos de vista que no incorporen las relaciones espaciales y temporales, la transversalidad, el carácter y calidad de las confrontaciones, la dignidad de los sujetos de conocimiento.

Palabras-clave: sujetos sociales, conocimiento social, pobreza, exclusión, diversidad, dignidad.

Recebido: $28 / 08 / 2005$

Aceite final: 21/11/2005 


\section{Exclusion and social knowledge}

\section{Raquel Sosa Elízaga}

Social sciences have undergone in recent years the confusion promoted from power about who social subjects are and how they behave. The proliferation of poverty, changes in social life, forms of struggle for survival have remained away from most investigators and politicians that apply programs oriented to distinct groups.

Social statistics have become along the years a sophisticated tool for the socalled social policies, whose aims have ranged from the claim to include the poor into the market to the contention of their movements through assistance schemes focused on potentially critical areas. Asking - and investigating - how many the poor are and where they are seems to be the newest and profitable way for politicians, officials, and experts.

Few are those who, from the historical and social perspective, have recognized the existence of collective behavioral codes that determine, beyond material living conditions, the existence of one or another form of government, of the specific policy applying to a social group, the movement of societies in the process of transformation.

Re-dimensioning the forms acquired by the social presence of the excluded in their diversity, its contradictory expressions, their demands, the exercise of their rights, is one of the major tasks of contemporary social sciences. It is a task that accepts no strict models, parameters, or structures of thought and categorization. It can hardly be thought as an individual work, but in no case it can be developed if one starts from viewpoints that do not incorporate spatial and temporal relations, transversalness, the character and the quality of changes, the dignity of knowledge subjects.

Key words social subjects; poverty, exclusion, diversity, dignity. 